\title{
EASY: Educational Alibava System
}

\author{
Carmen García ${ }^{1,}$, José Bernabeu ${ }^{1}$, Juan Herranz ${ }^{3}$, Carlos Lacasta ${ }^{1}$, Manuel \\ Lozano $^{2}$, Salvador Martí-García ${ }^{1}$, Giulio Pellegrini ${ }^{2}$ and Miguel Ullán ${ }^{2}$ \\ ${ }^{1}$ Instituto de Física Corpuscular-IFIC (CSIC-Universidad de Valencia) \\ ${ }^{2}$ Instituto de Microelectrónica de Barcelona-IMB-CNM (CSIC) \\ ${ }^{3}$ Alibava System \\ Corresponding author: Carmen.Garcia@ific.uv.es
}

\begin{abstract}
-
EASY, a plug-and-play educational system, is portable, compact and a complete system for micro-strip sensor characterization. Ideal for making basic or complex experiments. It is based on the Classic Alibava System [1], largely used within the CERN community to test micro-strip detectors for particle experiments. The system can be configured to work with pulsed laser light or radioactive sources.

The aim of this system is to illustrate students in the operation of a silicon strip detectors

The components of the EASY systems are the Control Unit and the Sensor Unit. The Control Unit is the heart of the system communicating with the Sensor Unit and the Computer software. It contains the Data Acquisition Control and it is also in charge of processing of the sensor data and trigger inputs. In addition, it contains an adjustable Hight Voltage unit for micro-strip sensor bias, with voltage and current display and includes the laser source. The Control Unit communicates with computer software via USB. The Sensor Unit accommodate a p-on-n silicon micro-strip sensor segmented in 128 strips.

EASY comes with an activity book where the students, through 10 exercises, are introduced in the main concepts and functionalities of micro-strip silicon detectors, used in the actual particle physic experiments. The book also provides a full description of the EASY device and the data Acquisition system.
\end{abstract}

Keywords - Education, ionization detector, micro-strip sensor, .data acquisition and processing, pedestal, noise, signal cluster, charge collection, depletion voltage, charge sharing, laser penetration.

\section{INTRODUCTION}

$\mathrm{E}$ ASY, the Educational Alibava System (EASY), a plugand-play educational system, is portable, compact and a complete system for micro-strip sensor characterization, ideal for making basic or complex experiments with silicon microstrip detectors. The students will be introduced in high-energy physics and particle detectors with hands-on experiences. They will be familiarizing with concepts such as MIP (Minimum Ionization Particles), charge collection, full depletion and charge sharing in strip detectors, among others concepts.

\section{THE EASY SYSTEM COMPONENTS}

The components of the EASY systems are the Control Unit (system's main board) and the Sensor Unit (containing the detector board), Fig. 1 shows the EASY components. The Control Unit is the heart of the system communicating with the sensor unit and the computer software. It contains the Data Acquisition Control and it is also in charge of processing of the sensor data and trigger inputs. In addition, it contains an adjustable High Voltage unit for micro-strip sensor bias, with voltage and current display and includes the laser source. This laser source is a pulse laser of $5 \mathrm{~ns}$ pulse width and a wavelength of $980 \mathrm{~nm}$. It is used to induce charge in the detector. The laser peak power is $0.6 \mathrm{~m} \mathrm{~W}$ and its spot around $20 \mu \mathrm{m}$. The Control Unit communicates with computer software via USB.

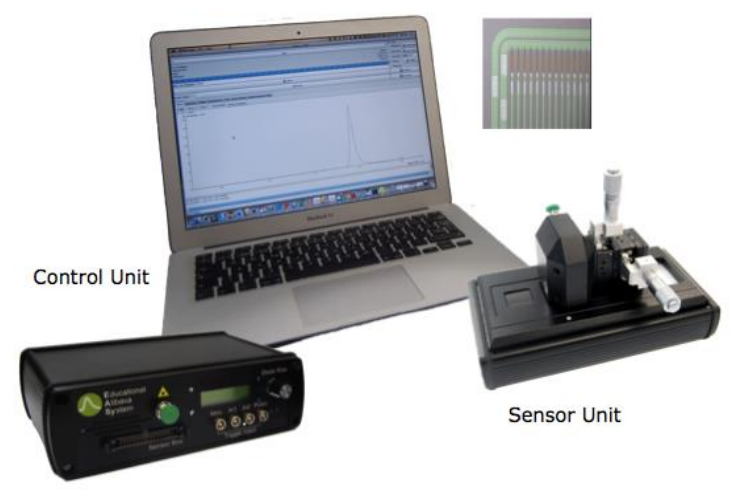

Fig. 1. EASY system with the Control Unit, the Sensor Unit and the acquisition program Alibava-gui running in the computer.

The Sensor Unit (Fig. 2) accommodate a p-on-n silicon micro-strip sensor, the same type of sensor used in actual ATLAS detector at LHC. P-on-n strip detectors consist typically of an around $300 \mu \mathrm{m}$ thick n-type bulk. On one side, a thin layer segments form strips out of p-type material, whereas the back implant is $\mathrm{n}+$. The abrupt junctions are formed between the strips and the bulk silicon. The depletion zone grows from the strips to the backplane so that, the device can operate partially depleted and the movement of holes forms the 
strips with a pitch of $160 \mu \mathrm{m}$. The Sensor Unit has a mobile part that slides over the box walls with horizontal and vertical micro-positioners screws. It has two positions to use the device with radioactive source or with laser light. In order to use it with radioactive source, the user will place the source on a carbon fiber rectangle aligned with the micro-strip sensor. When the device is use with the laser, the moving part of the Sensor Unit slides to focus the laser on the detector micro-strip sensor. The two micro-positioners allow to the movement of the laser lens in $10 \mu \mathrm{m}$ steps, horizontally and vertically, to focus the laser and to move the laser beam over the sensor, perpendicular to the strips.

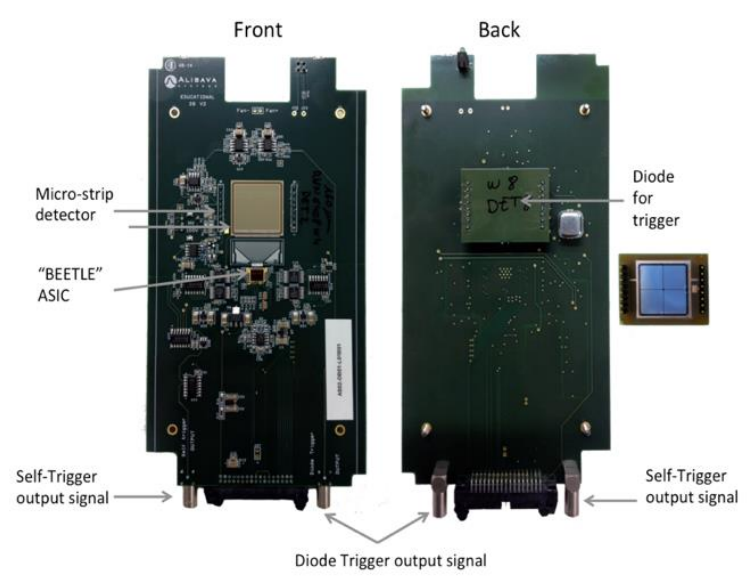

Fig. 2. Sensor Unit holding the micro-strip detector and the BEETLE in the front side and the Diode for trigger proposes in the back. The Board have two connectors for the trigger signal: Diode Trigger and Self-Trigger.

\section{PRINCIPLE OF OPERATION OF MIPS}

A MIP particle that traverses a silicon detector loses energy mainly by ionization. When the ionizing particle passes through the silicon lattice (see sketch on Fig. 3), this will tend to liberate a loosely bound valence electron generating an electron-hole pair. The free charge carriers formed by ionization in the silicon will move in the detector field in the depleted part of the detector. This is because the ionization in the depleted region is not recombined and the charges drift in the electric field. While in the non-depleted part, there is not electric field, charge will be recombined. Therefore, only the charge generated in the depletion part will contribute to the signal [3]. The width of the depletion zone is given by:

$$
d=D \sqrt{\frac{V}{V_{d e p}}}
$$

where $D$ is the detector thickness, $V$ de bias voltage applied to de sensor and $V_{\text {dep }}$ the voltage to reach full depletion. Thus, the charge collected of a silicon detector will be proportional to:

$$
\begin{array}{rr}
\sqrt{V / V_{\text {dep }}} & 0 \leq V \leq V_{\text {dep }} \\
1 & V>V_{\text {dep }}
\end{array}
$$

The signal generated in silicon detectors is generally of small amplitude, around 24000 electrons for full depleted $300 \mu \mathrm{m}$ sensor, hence amplification and shaping stages are required for its further processing. The read out and amplification of the signal of the detector is done by BEETLE chip [2], an ASIC (Application-Specific Integrated Circuit) developed for CERN LHC experiments.

\section{TRIGGER MODES}

The system incorporates three trigger modes: two asynchronized triggers (an external trigger and a self-trigger) and a synchronized trigger.

The external trigger, consists of discriminating a signal generated by a low noise silicon diode (Fig. 2 and 3). The silicon diode is mounted underneath the micro-strip sensor with a gap of few millimeters. When a particle crosses the microstrip and reaches the diode, the generated charge in the diode is discriminated to produce the trigger signal that starts the acquisition. The Diode Trigger mode is suitable for MIPs when studying the passage of particles through matter.

The self-trigger signal is generated by the fast comparator incorporated in the BEETLE chip. Each one of the 128 comparators has a different offset and response. Thus, an individual calibration of each comparator is needed in order to calculate the individual threshold value for each channel. With this calibration the a uniform for all channels is obtained. The EASY software provides an automated procedure to generate and calibrate all the thresholds. The self-trigger mode is convenient for acquiring data when the incident particles are completely absorbed, as it can be the case of photons.

Finally, the synchronize trigger consists in a pulse generated to trigger the laser, that should be synchronized before data acquisition.

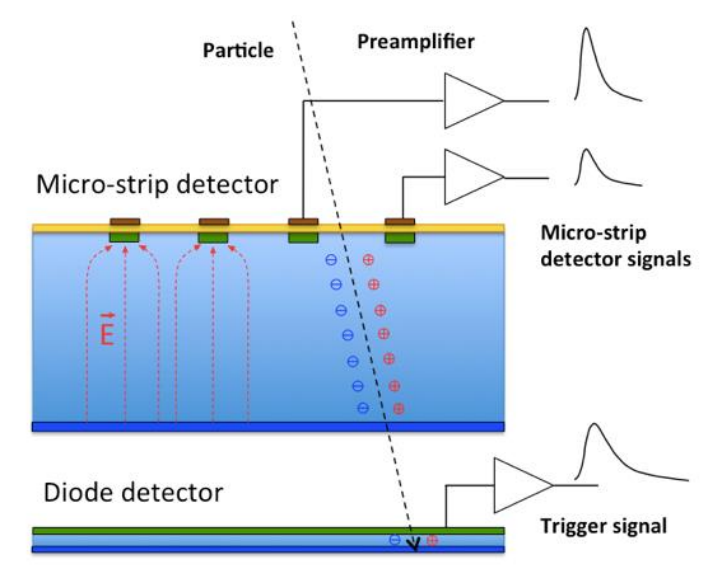

Fig. 3. Sketch of signal formation and the EASY diode trigger.

\section{DATA ACQUISITION AND MONITORING}

The software for data acquisition and monitoring (Alibavagui) is a graphical user interface, written in $\mathrm{C}++$, that controls the Control Unit. It is able to configure the sensor device, receive the data via USB bus and store it in a file for further analysis. Alibava-gui can be run in Windows, Linux or MacOs. 
This software also monitors the data during data acquisition, displaying parameter as: pedestal (ADC offset), electronic and common noise, gain, pulse shape, collected charge, single events per channel, etcetera. The software allow to demonstrate the basic principles of silicon sensor operation during the data acquisition, or, as in a real experiment, to record the data for a detailed analysis.

The system has five different run modes:

- Calibration: Alibava-gui programs the BEETLE chips to inject calibration pulses to all channels in order to characterize the electrical behavior of the ASIC. The calibration also allows obtaining the gain of the ASIC and the ADC to number of electron calibration curve.

- Laser synchronization: Alibava-gui sends a pulse that can be used to trigger the laser. This run mode scans the delay between the pulse sent by the Control Unit to the laser and the data acquisition.

- Pedestals Run: Alibava-gui generates an internal trigger that will allow computing the baseline for pedestals and its variation (i.e. the electronic noise).

- Laser Run makes a run where a laser beam injects the charge; one needs to run the laser synchronization mode beforehand in order to read back the optimal signal produced by the laser.

- Radioactive Source Run makes a run in which the acquisition is triggered by signals above the threshold in the input connectors suitable to work with radioactive source.

\section{SOME EXERCISES}

The EASY system illustrates the operation of a silicon strip detector with LHC readout electronics in 10 EXERCISES. Teachers can select the exercises according with the student level, from the basic principles of silicon detector operation to high level concepts on detector operation, electronics and data processing. Some of the aspects that can be covered are:

- Observation of electronic noise of a silicon strip detector as a function of bias voltage.

- Study of signal spectra due to MIPs crossing the silicon detector and the demonstration the Landau distribution shape of collected charge.

- Measurement of Charge Collection for different bias voltage and calculation of depletion voltage.

- Illustration of charge sharing between strip and relate this to the position resolution of the detector.

- Verification of the structure of a typical micro-strip detector.

- Comparison of the charge deposition of MIP particles crossing the detector and laser light stopping in the sensor bulk

- And, the calculation the laser penetration in the silicon.

Some of these exercises are described in detail below.

\section{A. Charge collection for a MIP particle}

The students can investigate the charge collected by a silicon micro-strip sensor when a MIP goes through it and triggering the diode behind the silicon sensor. The MIPs used are 0.546 $\mathrm{MeV}$ electrons irradiated by a ${ }^{90} \mathrm{Sr}$ source.
Before studying the charge formation in a silicon sensor, the student should understand the concept of pedestal, electronic noise (its dependence with the reverse voltage bias) and the common noise. The pedestal value of the ADC output is an effective offset. Namely, the pedestal value is the measured ADC counts when there is no signal at the input. In the EASY system the ADC offset has been set to the mean range of the ADC, around 500 ADC counts, to allow read positive and negative signals. The pedestal fluctuations are due to electronic non-uniformities of each strip. There are two sources of noise. The common mode shift or common noise, resulting for an electronic parasite pick-up which is specific of the chip and varies with time, fluctuating event by event. And, the electronic noise, defined as the fluctuation channel by channel. The common noise, the mean electronic noise and the pedestals are constantly monitored during the data acquisition.

Since the deposited charge can be collected in adjacent strips, a cluster algorithm has been implemented to integrated the charge deposition.
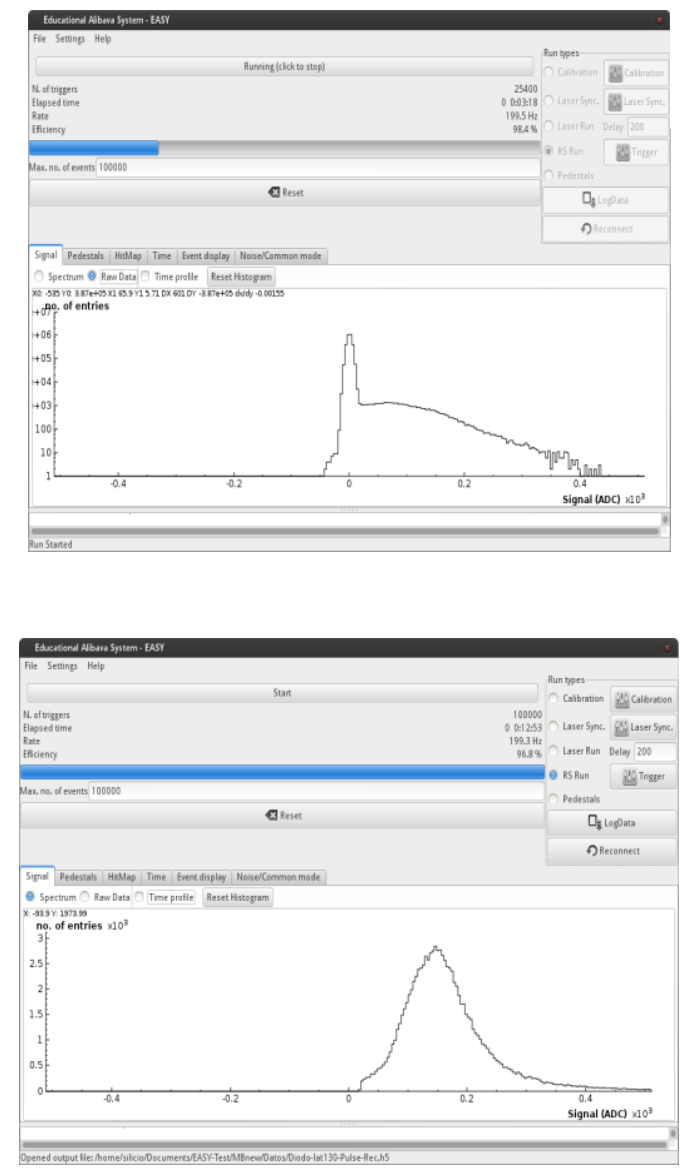

Fig. 4. Charge spectrum, in ADC count, of a Sr-90 source, (a) raw data in logarithmic scales and (b) after data analysis.

Fig. 4 (a) shows, in logarithmic scale, the accumulated charge (raw data) during the data acquisition. The large pick corresponds to electronic noise of the channel without charge deposition and the tail contain the collected charge. After data analysis (Fig. 4 (b)), one can get the charge spectrum showing 
an asymmetric distribution. As mention in section III, when the ionizing particle passes through the silicon he lattice, this will tend to liberate a loosely bound valence electron generating an electron-hole pair. Occasionally the ionizing particle will interact with a more tightly bound electron than the silicon valence electrons and, as a result, will create an electron with a significant amount of kinetic energy. Due to these rare highenergy events, the energy spectrum of the deposited energy in the silicon detector is a non-symmetric distribution. The distribution could be fit by a Landau function convoluted with a Gaussian distribution, and obtaining the more probably charge from the peak of the distribution. Notice that the more probable charge differs from the mean charge (the more probable charge is 0.7 times the mean charge). After calibration (converting ADC counts in number of elections) and considering the average energy neeed to produce a single electron-hole in silicon, one can obtain that the mean energy deposition of a MIP in $300 \mu \mathrm{m}$ is around $110 \mathrm{KeV}$.

Since only the charge generated in the depletion part of the sensor contributes to the signal. Varying bias voltage, the students can observe how the charge spectrum variate proportionally to the deplete area of the sensor. By measuring the more probable charge for different depletion voltages, they can obtain the characteristic curve of charge collection as a function of the bias voltage (equation (2)). Fig. 5 shows how the charge collected grows with bias voltage till a plateau is reached when the sensor is fully depleted. A bias voltage over $80 \mathrm{~V}$, is needed for full depletion.

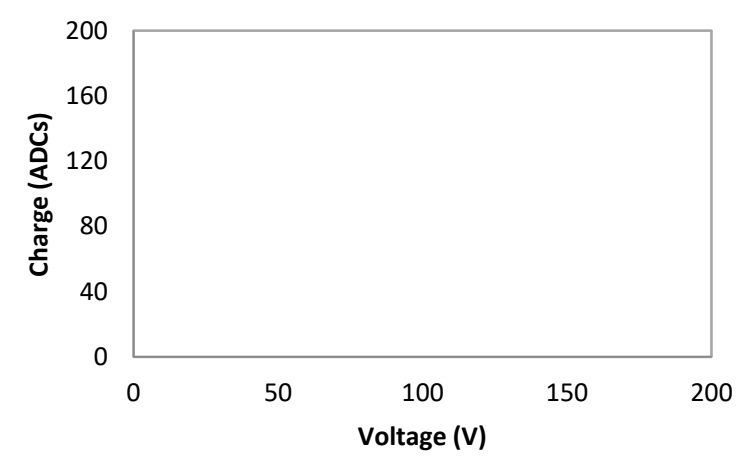

Fig. 5. The plot shows how the charge collected, in ADC counts, increases with bias voltage till a plateau is reached when the sensor is fully depleted.

\section{B. Strip Structure and Charge Sharing}

EASY system incorporates a 980 nanometers pulsed laser, to induce charge in the micro-strip sensor, mimicking the incidence of a charged particle in the detector. Two micropositioners screws allow to movement the laser lent in 10 micro-meter steps, horizontally and vertically. The vertical micro-meter screw is used to focusing the laser while the horizontal screw will allow to move the laser bean across the strip. One should be aware that laser beam is reflected in aluminum strips. So, if the laser beam insides over the strip no charge will be collected.

Starting for an initial incident position of the laser beam, for instance over strip 40. One can measure the mean charge in the channel 40 and the adjacent strips, strip 39 and 41 . In this initial position since laser beam is reflected in the aluminum strip a charge compatible with the electronic noise should be observe for all strips. When the laser moves away from the center of the

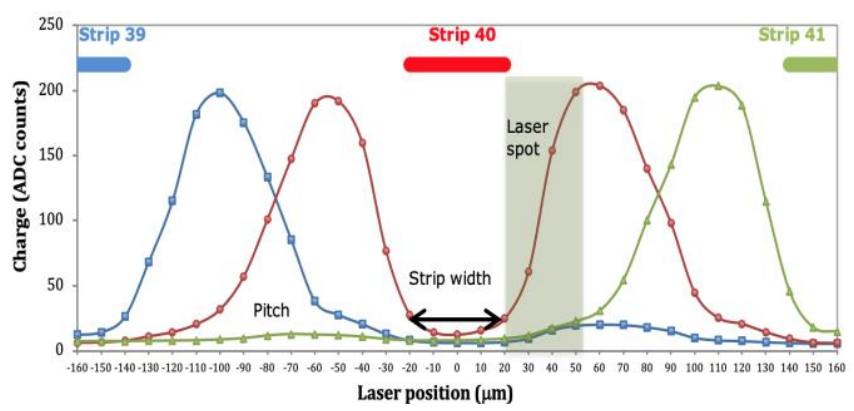

Fig. 6. Signal deposition by the laser bean in three consecutive strips obtained by moving the laser in $10 \mu \mathrm{m}$ steps perpendicular to the strips. The signal recorded by strip 39 is represented in blue, in red the signal in strip 40 and in green the signal in strips 41.

strip 40 to strip 41 , a small amount of charge begins to be collected in the strips 40 . This is due to the fact that part of the incident light is still reflected on the aluminum. keeping moving the laser beam, the charge on strip 40 will increase. When the beam falls between the strips, the charge will be shared between strips 40 and 41 . If we continue moving forward, strip 41 will start collecting more charge till reach its maximum. On the other hand, the charge collected by strip 40 will degrees till became compatible with electronic noise. We will continue this process moving the laser, right and left, for the initial position, towards strip 41 and towards strip 39.

By plotting the mean charge collected by three adjacent strips, showed on Fig. 6, we can illustrate how the charge is shared between strips and prove the strip structure. Also, to determinate the strip width, for the laser path without collected signal collected. Moreover, the beam spot will be measuring from the distance between the position when the laser beam starts generating charge in the detector and the maximum of the charge. And finally, the micro-strip pitch can be measure as the distance between two minima of collected charge as shows in Fig.6.

\section{Laser beam and MIP particles}

Using the laser beam, the growing of the depletion zone with the bias voltage and the laser penetration depth, can be study, by recording the charge collected for various bias voltages. The measured charge will be a convolution of the extension of the depletion zone and the energy deposition within the silicon.

In the case of an incident MIP, the energy deposition is uniformly in the silicon bulk, therefor the measured charge is proportional to depletion voltage till full depletion is reached as expressed in equation (2). While for a laser beam, the energy deposition is exponential and given by:

$$
E(y)=E_{0}\left(1-e^{-y / a}\right)
$$


where $y$ is the penetration distance of the laser and $a$ is the stopping distance (penetration distance). The stopping distance depends on the laser energy or equivalently the laser wavelength. Therefore, for laser light, the charge collection will depend on the depth of the depletion zone but also on the energy lost (i.e. on the laser wavelength). The higher the wavelength of the laser the faster the raise of the charge collection with the bias voltage to reach the plateau [4]).

The penetration of laser in silicon has been measured for some wavelengths, for example, for the $960 \mathrm{~nm}$ the penetration is around $74 \mu \mathrm{m}$ and for $1037 \mathrm{~nm}$ is $380 \mu \mathrm{m}$ [5]. The laser of the EASY system has a wavelength of $980 \mathrm{~nm}$, a priori; its penetration in silicon is unknown.

The Charge Collection Efficiency (CCE) is defined as the fraction between the measured charge for a certain bias voltage and the charge when the detector is fully depleted. For an incent laser light beam the $\mathrm{CCE}$ as a function of the bias voltage $(V)$ is given by:

$$
\operatorname{CCE}(V)=\frac{\left(1-e^{-d(V) / a}\right)}{\left(1-e^{-300 / a}\right)}
$$

Where $d(V)$ is the width of the depletion area as defined in equation (1) and can be calculated for each bias voltage $(V)$ from the exercise with radioactive source. Fig. 7 represents the CCE for different laser penetrations $(a)$, together with the CCE for MIP as measure in the radioactive source exercise. For laser penetration larger than $1 \mathrm{~mm}$ the laser data is a good approximation to a MIP.

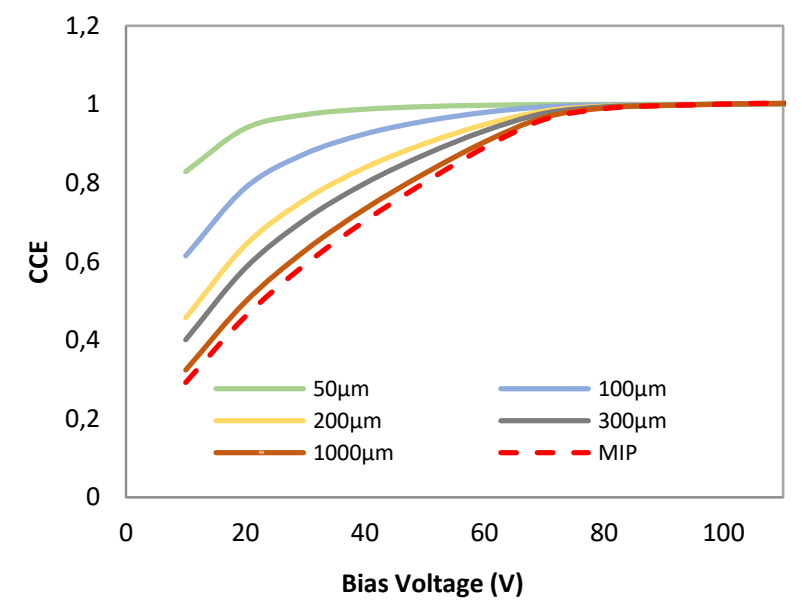

Fig. 7. Theoretical CCE for different laser penetrations and measure CCE for a MIPs.

By measuring CCE with the laser beam for different bias voltages and fitting the obtained curve using equation (4), one can obtain that penetration of a $980 \mathrm{~nm}$ wavelength laser, the valuer obtain is $167 \mu \mathrm{m}$. Fig. 8 shows the fitting curve and the MIP curve for comparation.

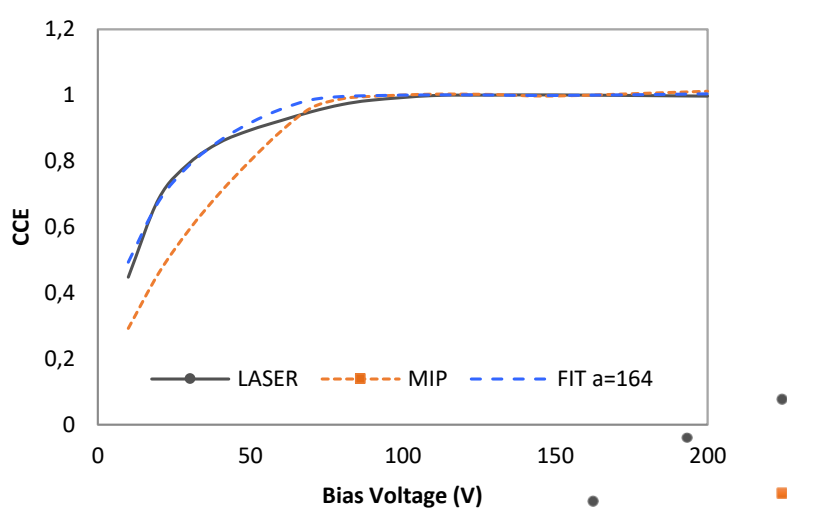

Fig. 8. Charge Collection Efficiency from laser data, fit to a penetration length of $167 \mu \mathrm{m}$ and data for a MIP electron calculated in EXERCISE 6 for comparison.

\section{Spectroscopy using the self-trigger}

The BEETLE ASIC incorporates a self-trigger signal that will be issued whenever one of its 128 channels registers a signal larger than a preprogramed threshold. To accomplish that, the incoming signal is split into two branches. One goes through a shaper and then to a comparator that will issue the trigger signal for values above the threshold. The signal of the second branch is used to measure the generated charge in the detector.

The self-trigger mode allows measuring the charge induced by particles crossing the detector but also photons or alpha particles absorbed in it. The range of the ADC incorporated in the Control Unit and the dynamic range of the BEETLE define the upper energy limit the system can measure. While the lower energy limit is defined by the noise of the system. Typically, the energy range goes from $20 \mathrm{KeV}$ to $300 \mathrm{KeV}$.
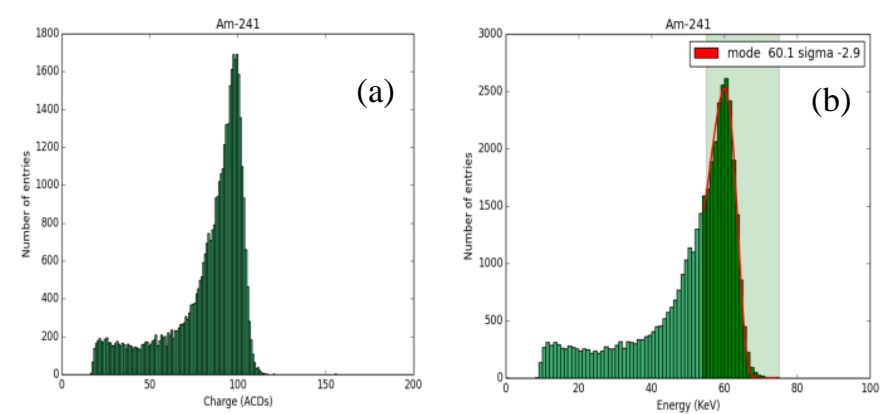

Fig. 9. Charge collected from Am-241 encapsulated source in ADC counts (a) and in energy (b).

As an example, Fig. the collected charge from an ${ }^{241} \mathrm{Am}$ encapsulated source in ADC count (a) and after energy calibration (b). Since the encapsulation of the source absorbers the beta emersion from the ${ }^{241} \mathrm{Am}$, only the $60 \mathrm{KeV}$ gamma rays reach the sensors. Fig. 9 (b) show clearly the gamma emission piek. 


\section{CONCLUSIONS}

EASY is a complete instrumentation system dedicated to Silicon micro-strip Radiation Detectors, representing the stateof-the-art in detector characterization using the same technologies that the actual LHC strip detectors. The aim of this system is to illustrate the operation of a silicon strip detectors, ideal for making basic or complex experiments with silicon micro-strip detectors according with the student level. A complete data acquisition and monitoring software allows to use the system for demonstration of the principles of micro-strip detectors operation. Also, the data can be downloaded for a full analysis as in a real experiment.

The "Activity Book For Students" provides a full description of EASY device, data Acquisition system and 10 exercises to guide the student in the basic principles of micro-strip silicon sensors. The system is commercialized by ALIBAVA SYSTEMS (www.alibavasystems.com), under license of the Spanish National Research Council (CSIC) and the University of Valencia (Spain).

\section{REFERENCES}

[1] ALIBAVA Collaboration. "A portable readout system for microstrip silicon sensors”. IEEE Trans.Nucl.Sci. Vol. 56 (2009): 1642-1649.

[2] Löchner, S y Schmelling, M. "The Beetle Reference Manual - chip version 1.3, 1.4 and 1.5". Max Planck Inst.: LHCb-2005-105.

[3] Lutz, G. "Semiconductors Radiation Detectors, volume 2nd Edition". Springer, 1999.

[4] Abt, I. et al. "Characterization of Silicon Microstrip Detectors Using an Infrared Laser System”. Nucl. Instr. Meth A423 (1999): 303-319.

[5] Lide, D. R:. "Handbook of Chemistry and Physics". Editor-inChied, 77th Edition, 1996. 